\title{
Rule of Thirds Based Fast Color Error Evaluation for QoE- Guaranteed Mobile Media Services
}

\author{
Tan Loc Nguyen ${ }^{1}$, Jinsul Kim ${ }^{1 *}$, Yonggwan Won ${ }^{1}$ and Sang-huyn Park ${ }^{1}$ \\ ${ }^{1}$ Department of Electronic and Computer Engineering, \\ Chonaman National University \\ 500-757 Gwangju, Korea \\ nguyentanloc03200@gmail.com,jsworld@jnu.ac.kr_(*corresponding author) \\ Abstract
}

Video streaming has become one of the most essential ways in online communication. While video streaming is widely used in most of the Mobile Multimedia applications, it is not only necessarily required to maintain the high video quality bu also minimized the video processing time to ensure the Quality of Service $(Q \circ S)$ as well as Quality of Experience (QoE) of the system in live broadcasting. Thispaper introdices the different way to evaluate the transmission errors in video applications by measuring the error with un-uniform distribution of information importance factor in parels to achieve a suitable order in error correction. By changing the QoS evalwating method by considering Regions of Interest, especially fundamental rules inart study such Rule of Thirds, QoE should be improved to get closer to the viewer concerns and increase thesystem efficiency. Error detection as well as correction should be significantly corrested in priority orders.

Keywords: Rule of Thirds, QoE, QpS, Mobile Media Application, Processing Time

\section{Introduction}

Recently, with rapid growth of the Internet, streaming multimedia has not only become very popular but also the nost bandwidth consumption in the Internet. There were many video services such as HWTV, Video on Demands (VoD), Video conferences, Voice over IP (VoIP), IPTV, Mobile TV and their additional services, which usually took more than half world wide bandwidth of the Internet (55\% in 2012) and 69\% in 2017 [1]. The standards for video quality in multimedia delivery were also a serious concern. Some researches and methods have been done to evaluate the quality of transmitted video in order to guaranty QoS. The QoS is usually calculated by the mismatch from the original and the end user received multimedia in the reference measuring methods or logical self-inferring techniques in the no reference methods [2]. Generally, the errors, in any kind of data, could be caused by source encoding, compression, network encoding and etc. Even in the best systems, errors should always exist. Therefore, to maintain a good QoS, errors should be corrected.

However, the more careful the errors are corrected, the longer time is required. Therefore, only the important errors should be corrected before considering the other less important one to ensure the minimum processing time even though some errors would exist in the system. In fact, in most of the kinds of multimedia, some errors are still accepted by users. The "JPEG" image compression, "MP3" audio encoding and even the "MPEG" video are the obvious examples for acceptable error definitions. 
It is very necessary to manage the processing time in live multimedia streaming. Moreover, the acceptable losses or errors could reduce the complexity of system. Actually, there is constrain for human abilities in recognizing visual errors during experiencing single pictures, especially in videos where errors occurs really fast. In fact, some errors locating in unimportant regions in video frames could be ignored.

According to the famous rule in art, "Rule of Thirds", the fundamental rule in photography and filming, the human attention in image or frame is not uniform in every region [4]. There are some most focusing regions and some others are not. That rule stated that if an image is divided into three parts by two parallel lines in each dimensions, the most subjectively important regions should be the four intersections as well as the four lines. As the results, any error in these regions could cause serious losses in pixels' information, which means directly affecting the main contents and easily be realized. A higher priority in detection and correction should be done for these errors. On the other hand, the viewer seldon focuses in some errors appearing in corners of screen, and sometimes these êrrors eould be omitted. Due to that reason, detecting and calculating such errors in video could be improved by being carried on with the Rule of Thirds as un-uniform important factor distribution in pixels over whole single video frame.

In this paper, the overall color error would be subjectively measured with different priority coefficients for different regions of interest (ROI) such (as intersections, dividing lines, neighbors dividing lines, and corner regions Color errors would be evaluated by the color quality metric with HSI transformation noder [5]. As the result, the important regions would expectedly cause more serious effects and higheroverall errors in video quality metric. This approach would not only be applied to color error in video quality but also for other video features such as block distortion, blurring, ete. In general, this method would be basic steps for the new subjective evaluation of video quality with art's rule applications.

The next parts would demonstrate how to implement the Rule of Thirds in color measurements as well as its tests and results for the methods.

\section{Techniques}

\subsection{Quality Monitoring System}

While no reference method at this time still has some limitations, it is possible to evaluate this project with the reduced reference method to measure the color errors in each video frames. Generally, it is necessary to get original and transferring videos as the broadcasting server and end-user respectively. The errors could mainly be created in transmission and evaluated by measuring the difference between these two videos.

\subsection{Color Model for Evaluation}

When color error is the main target in quality assessment, it is necessary to represent the color as a model of assessment. Generally, any color is represented by three main components: red, green and blue (RGB). Any change in value components will introduce a difference in visual color. The set of colors would come from black (when all three values are 0 ) and white (when all three values are 255). However, the changing in color in this model is nonlinear and is not a good logical representation for colors implementation.

Instead of using RGB model, there is another color representation system, called HSI (Hue - Saturation - Intensity) model [5-[7]. The HSI model is a better color representation to the human vision than RGB. There are three color components in this model: Hue, Saturation and Intensity. In detail, Hue is a feature representing how pure a color is or which color is most 
similar to, while Saturation describes how a color looks like the pure color under the effect of white light [5].

In this technique, the overall colors would be used to compare with the reference over whole every video frames [2]. However, there are the coefficients of interest when calculating errors either in RGB or HSI models. Firstly, it is necessary to convert RGB into HSI model as following, (assuming R, G, B as the average values):

$$
\begin{aligned}
& I=\frac{1}{a}(R+G+B) \\
& S=1-\frac{a}{(R+G+B)}\left[\min \left(R_{x} G_{x} B\right)\right] \\
& H=\cos ^{-1}\left\{\frac{\frac{1}{2}((R-G)+(R-B)]}{\left[(R-G)^{2}+(R-B)(G-B)\right]^{1 / 2}}\right\} \\
& \text { If } \left.S=0, H \text { is meaningless If }(B / I)>\left(G_{I}\right)=H=360-H\right\} .
\end{aligned}
$$

In order to calculate color error, every pair of video frames, should be used to measure these color components and return the different vector between them. Let the Saturation and Hue be $S_{1}$ and $\theta_{1}$ in the original and $S_{2}$ and $\theta_{2}$ in transferred video frame respectively. The representation for these components is shown in Fig 1 and Fig 2 . Let's define " $d$ " as the difference vector in color between two frames, as the colorerror. By applying the Cosine's law in triangle, the below equation was used to calculate the color error in the two corresponding frames.

$$
d=\sqrt{S_{1}{ }^{2}+S_{2}{ }^{2}-2 \times S_{1} \times S_{2} \times \cos \left(\mid \theta_{2}-\theta_{1} l\right)}
$$

According to the characteristics of HSI color model stated by Gonzalez and Woods[5], the Intensity component, $\mathrm{I}$, is decoupled from the color information in the image. That is only meaningful for image computer based processing. The other two components $\mathrm{H}$ and $\mathrm{S}$, on the other hand, are intimately related to the way in which human being perceive colors. Therefore, for the simplicity of processing, only Hue and Saturation would be considered in this paper to evaluate the color error in frames. The procedure was described in equation 1,2 , 3 and 4.
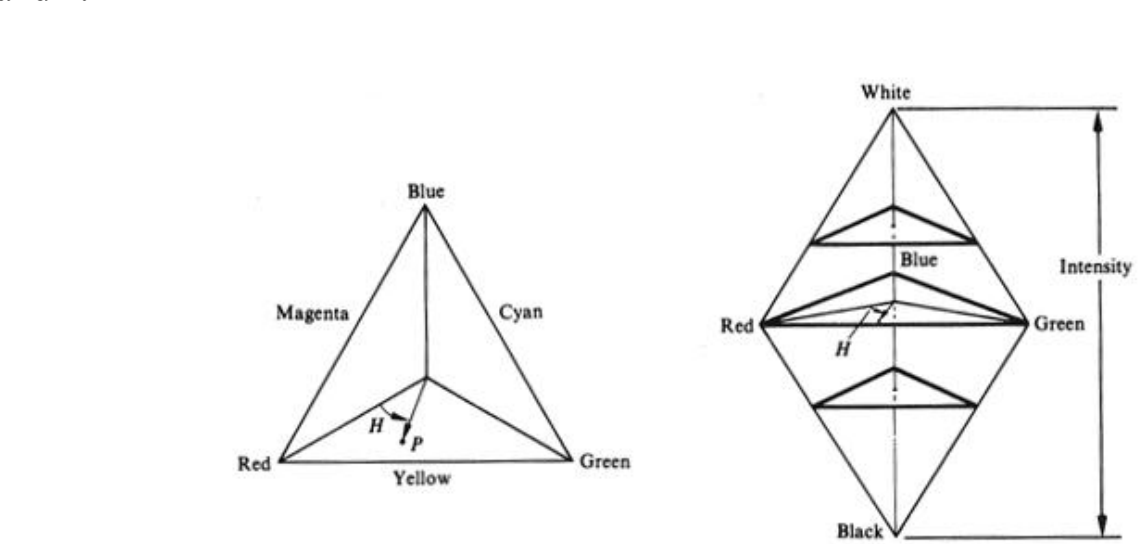

Figure 1. HSI Color Model with Hue-Saturation and Hue-Saturation-Intensity 


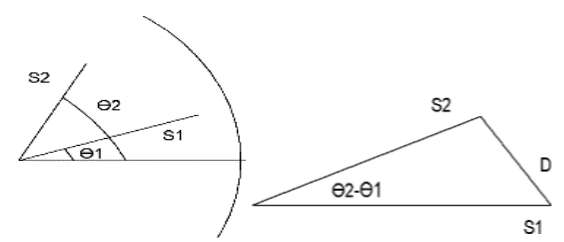

Figure 2. Geometric calculating model of Hue-Saturation-Intensity in HSI

\subsection{Regions of Interesting - Rule of Thirds}

Normally, every pixel in both frames would be equally compared. Nevertheless, this consideration might not be corrected when it takes into account the acceptable errors unexploited by users. In fact, there are some regions would caught more viewdr's attention while some would not. Therefore, the frame should be divided into several ROIs with various priority factors. Because of the important of information loss in type 4, these regions would have the highest coefficient of priority factor for error calculating. For higher error evaluating resolution and according to the Rule of Thirds [4], the frame is actually divided into 12 parts with fixed two dividing lines in each dimension. The distribution is shown in Fig 3. There are coefficients as $0.03,1.2,2.5,6$ for Type $1,2,3$ and 4 respectively. Then, the color quantity in each video image would calculate as (6), (7).

In the below equations, $r, g, b$ are the color components inside each pixels and $R_{i}, G_{i}, B_{i}$ are an average value of color components it each regions There are four values for "i" as the four types of regions. The " $\mathrm{j}$ " indicates the frames in pair, 1 is original and 2 is transferring video. The smallest rectangle unit in a) frame was called unit-block whose dimensions were calculated as frame height $/ 12$ and frame_width/12. As the result, the Area of type I, type II, type III and type IV would be 16, 48, $\overline{64}$ and 16 unit blocks, respectively. Afterward, it is necessary to provide an average $\mathrm{R}, \mathrm{G}$, and B for whole frame with the priority coefficients for each regions as $(8),(9)^{\circ},(10):$
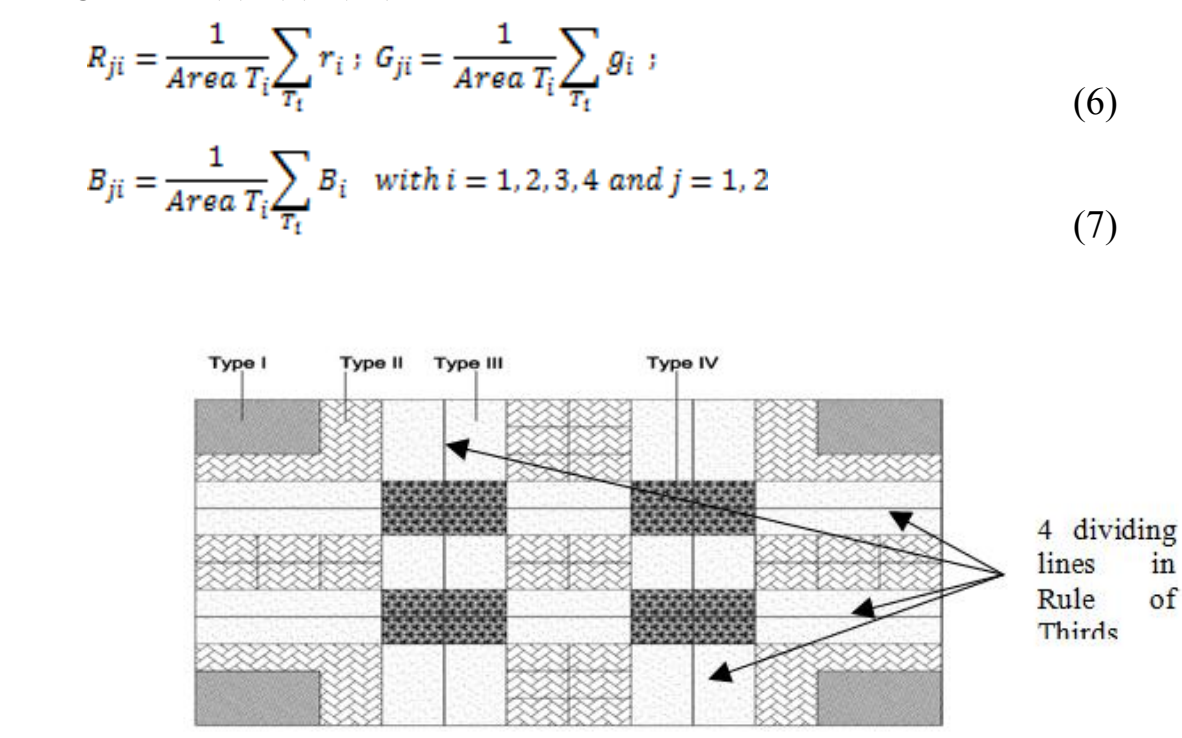

Figure 3. Rule of Thirds division in Frame

$$
\begin{aligned}
& R_{j}=0.03 \times R_{1 j}+1.2 \times R_{2 j}+2.5 \times R_{a j}+6 \times R_{4 j} j^{n} \\
& G_{j}=0.03 \times G_{1 j}+1.2 \times G_{2 j}+2.5 \times G_{a j}+6 \times G_{4 j^{3}}
\end{aligned}
$$




$$
R_{j}=0.03 \times B_{1 j}+1.2 \times B_{2 j}+2.5 \times B_{3 j}+6 \times B_{4 j} j^{3}
$$

\section{Experiments and Results}

\subsection{Objective Test}

In this experiment, there are two samples clips. One of them has been created as the transmitted videos by adding color errors in some particular regions in original frames. Generally, the clip contained 130 frames in total and the errors have been added in every period of 21 frames. It is necessary to have the same number of errors for each of frame regions.

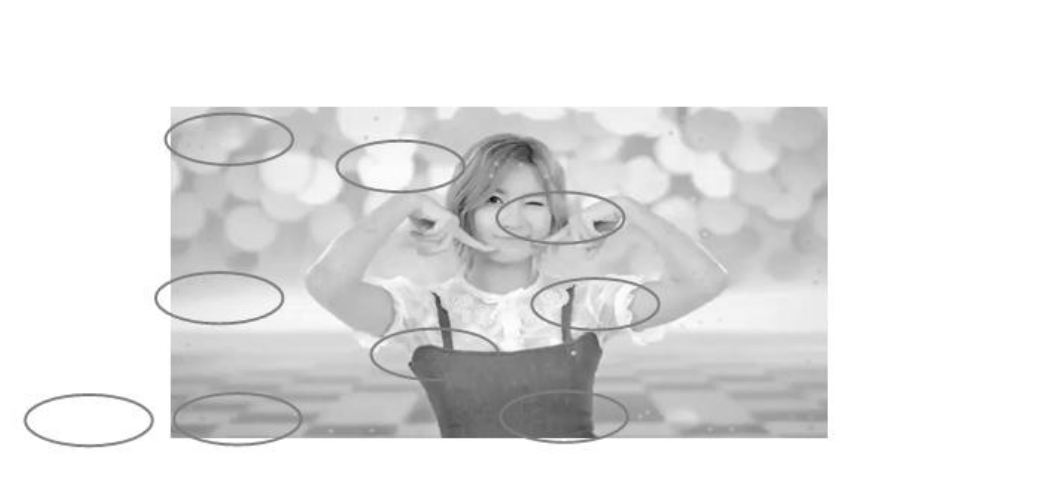

\section{Figure 4 , One of êror trames}

Firstly, to ensure the correct performanee of the evaluating system, same clips have been used to ensure the error free result and guarantee normal performance, as Fig 4.

Secondly, the 48 color error sample videe would be used as transferred video while original video was remanded. In the Fig 6, the continuous line (resulted by rule of Thirds algorithm) indicates the serious color errors in the Rule of Thirds regions (at the second half of the video), which would eause much impacts to the main content on the screen. The others errors would cause less effects to the overall video quality. However in the dash line (representing the normal error evaluation, the error would be nearly the same for all the regions.

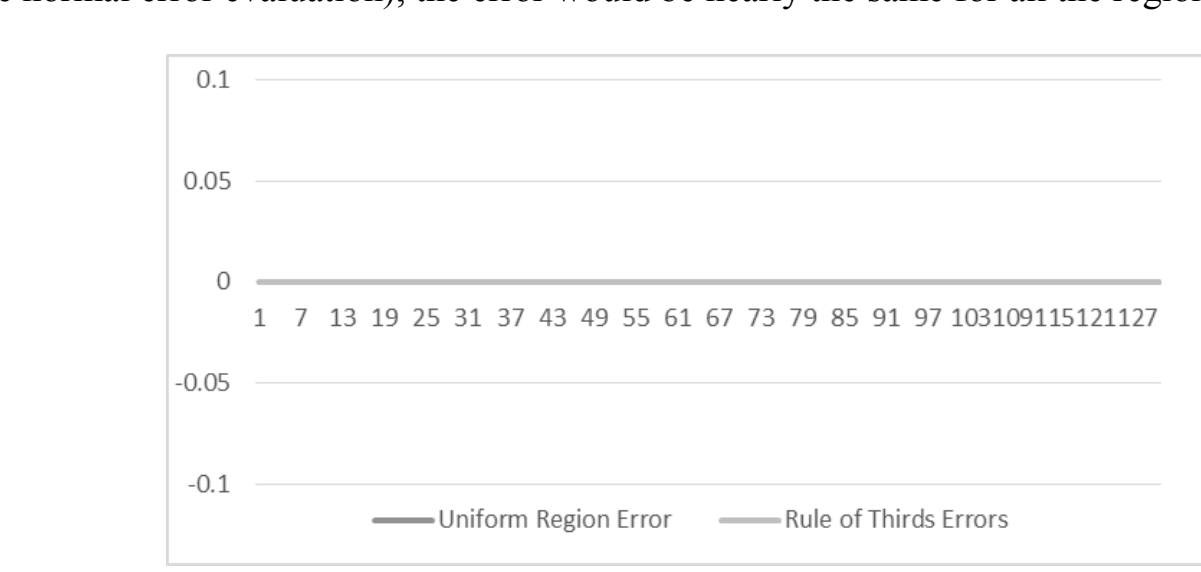

Figure 5. Same video samples testing result 


\subsection{Subjective Test}

Three sub-tests, Rule of Thirds regions, Viewer Opinion and removing Rule of Third errors tests, have been carried out. The Rule of Thirds regions test demonstrates the sensitivity of viewer to color errors and their locations. The same clip sample has been reused in this test. The clip was played together with the original clip to avoid the effects by serial error frames. There are 15 observers were asked to relatively notice error locations while focusing on the main contents of the video sample. Fig 5 shows the total number of errors have been noticed in each regions. In details, there is a few of errors could be realized the corners of screen (the region type I). With the same number of errors added, the recognized errors increased in the frame margin (type II), especially in the Rule of Thirds regions (type III and IV). Even though, the number of errors is decreasing in the-intersection regions, the error density is still highest. This means whenever the errors takes place in this region, it would be easy to be noticed by viewers.

Secondly, viewer opinion tests give a clearer view of different regions? impacts in viewer satisfaction with each of error. In this test, for each of the errot's location (done in previous test), it is required for observers to give the error' satisfaction/Invery Mean Opinion Score (MOS) has been used, in which viewer's dissatisfaction with 6 is aceeptable, and 5 is highly uncomfortable for every appearing error. In the Fig 6, the gray areas indicate the dissatisfaction level (Invert MOS). This quantity is changedpproportional to the changes in Rule of Thirds region error measurement. With the same namber of errors, the QoE in the Rule of Thirds region has been affected much more than the others. Therefore, it is necessary to correct these errors first to maintann the QoK coefficients. In other words, the quality in these interesting regions should be ensured for QoE Guaranty.

Finally, most of the errors in Rule of Third segions (type III and IV) have been removed. Most of the observers realized that the quality has strongly been increased and there was no complain for video quality. Few of the viewer still realized the others errors. The other regions result was also been affected due to viewer's mistakes in realizing error locations at the first stage.

Note that for all the test including objective and subjective tests, for getting the better and closer to real conditions of live broadcasting, the test was played quince (4 times) only to ensure the observers would not try to detect the errors instead of enjoy the contents.

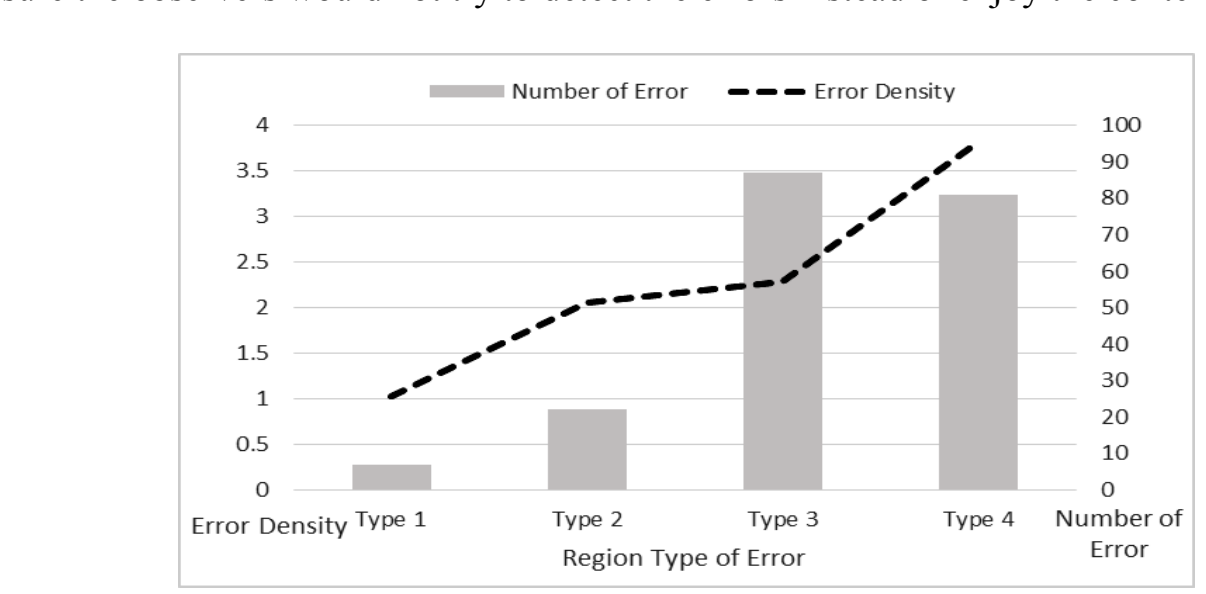

Figure 6. Subjective testing result in Regions of Interesting 


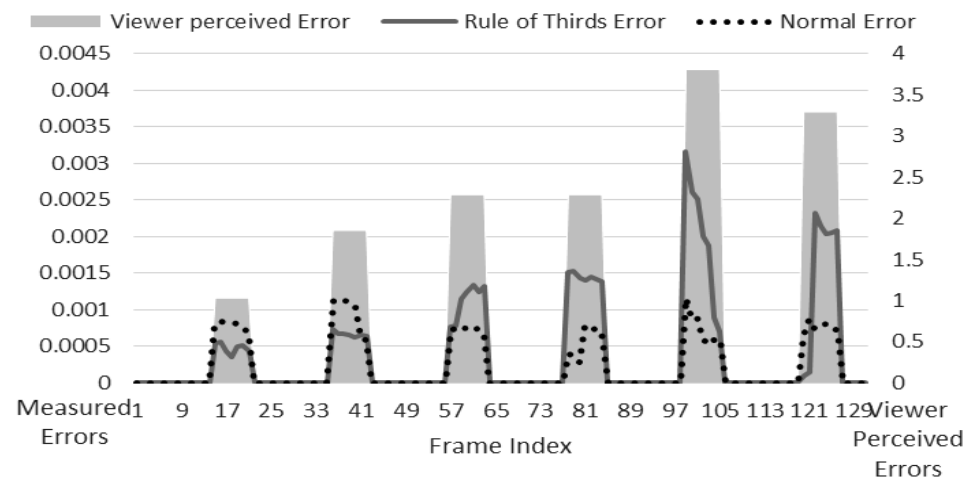

Figure 7. Testing results

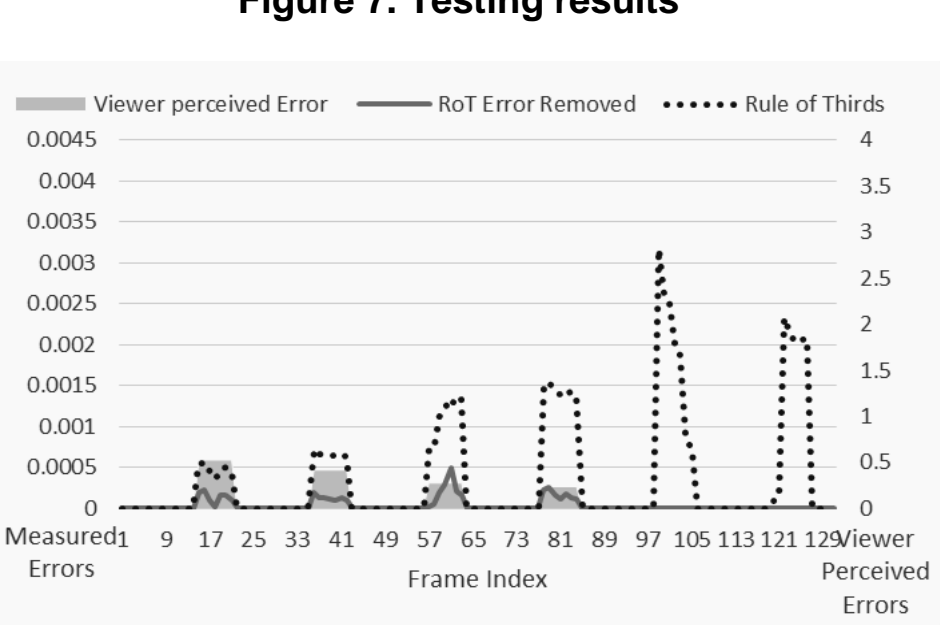

\section{Conclusion}

Figure 8 Rule of Thirds Error Removed.

The video quality, one of the most important concerns in streaming multimedia system, should not only be carnice out by computing the overall error factors but also based on viewer perceived experience. In fact, Rule of Thirds including some viewer's interesting regions rules could be applied to preciously adjust the QoE measuring method and simplify the system structure as well as analytical algorithms. Even though different kinds of rule can give different prority in error measurement, this Rule of Thirds as the fundamental rule in Art study could give the basic improvement and acceptable results for color error evaluation and measurement. In general, this technique can be applied to most kinds of error managements when the priority of error correction is highly required.

\section{Acknowledgments}

"This research was supported by the MSIP (Ministry of Science, ICT and Future Planning), Korea, under the ITRC (Information Technology Research Center) support program (NIPA-2013-H0301-133005) supervised by the NIPA(National IT Industry Promotion Agency)". 


\section{References}

[1] Cisco System Inc, "Cisco Visual Networking Index: Forecast and Methodology”, White Papers, 2012-2017, (2013) May.

[2] M. A. Hasan, W. J Kim, C. G. Kim, J. S. Kim, H. W Lee and W. Ryu, "Color Error Measure for IPTV Service Quality Evaluation”, ICACT 2008, ISBN 978-89-5519-136-3, (2008).

[3] L. Ze-Nian and S. D. Mark, "Fundamental of Multimedia", Pearson Education International, (2004), ISBN: 013-127256-X

[4] J. Hart, "The Art of the setoryboard: a Filmmarker's Introduction", 2nd edition, Focal Press, (2008), ISBN: 978-0-240-80960-1.

[5] R. C. Gonzalez and R. E. Woods, "Digital Image Processing", Prentice Hall, ISBN: 0-201-50803-6. Chap 4, (1993), pp. 221-245.

[6] M. Sonka, V. Hlavac and R. Boyle, "Image Processing, Analysis, and Machine, Vision", 2nd Edition, PWS publishing, Chap 2, (1999), pp. 25-27 and pp. 35.

[7] C. L. Chien and D. C. Tseng, "Color Image Enhancement with Exact HSI Color Model', ICICInternational, (2011), ISBN: 1349-4198.

[8] J. S. Kim, T. W. Um, W. Ryu, B. S. Lee and M. S. Hahn, "Heterogenteous Networks and Terminal-Aware QoS/QoE Guaranteed Mobile IPTV Service", IPTV Systems, Standards and Architectures: Part II, IEEE Communications Magazine, (2008) May.

[9] K. Seshadrinathan, R. Soundararajan, A. C. Bovik and L K. Cormack, Study of Subjective and Objective Quality Assessment of Video", IEEE Transactions on Image-Processing, (2009).

[10] C. Fenimore, V. Baroncini, T. Oelbaum and T. K. Tan "Subjective Testing Methodology in MPEG video cerification", National Institute of Standards and Technology -'Gathersburg MD, Fondazone Ugo Brdoni, Institute for Data Processing - Munich University of Technology, NTT DoCoMo Inc - Multimedia Laboratories - Yokosuka.

[11] B. Gardlo, M. Ries, M. Rupp and R. Jarina, "AQ E Evaluation Methodology for HD Video Streaming using Social Networking".

[12] I.T Young, J. J. Gerbards and L. J. V. Vliet, "Fundamentals of Image Processing", 2nd Version, Delft University of Technology, (1998), ISBN: 90-7569-(10-7.

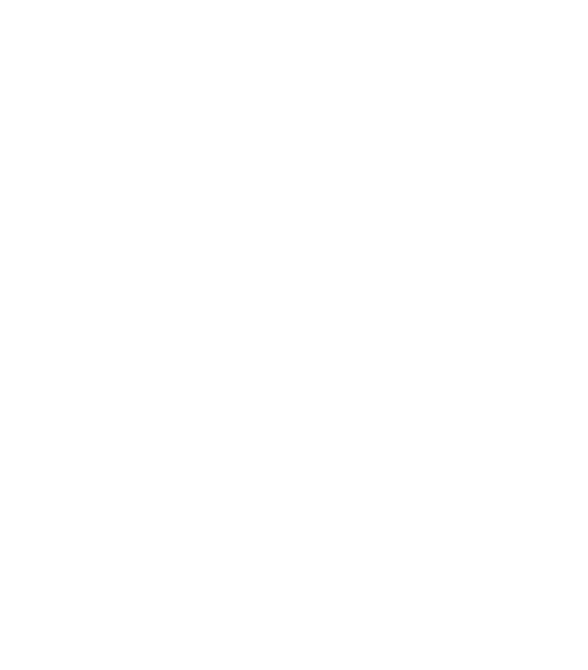




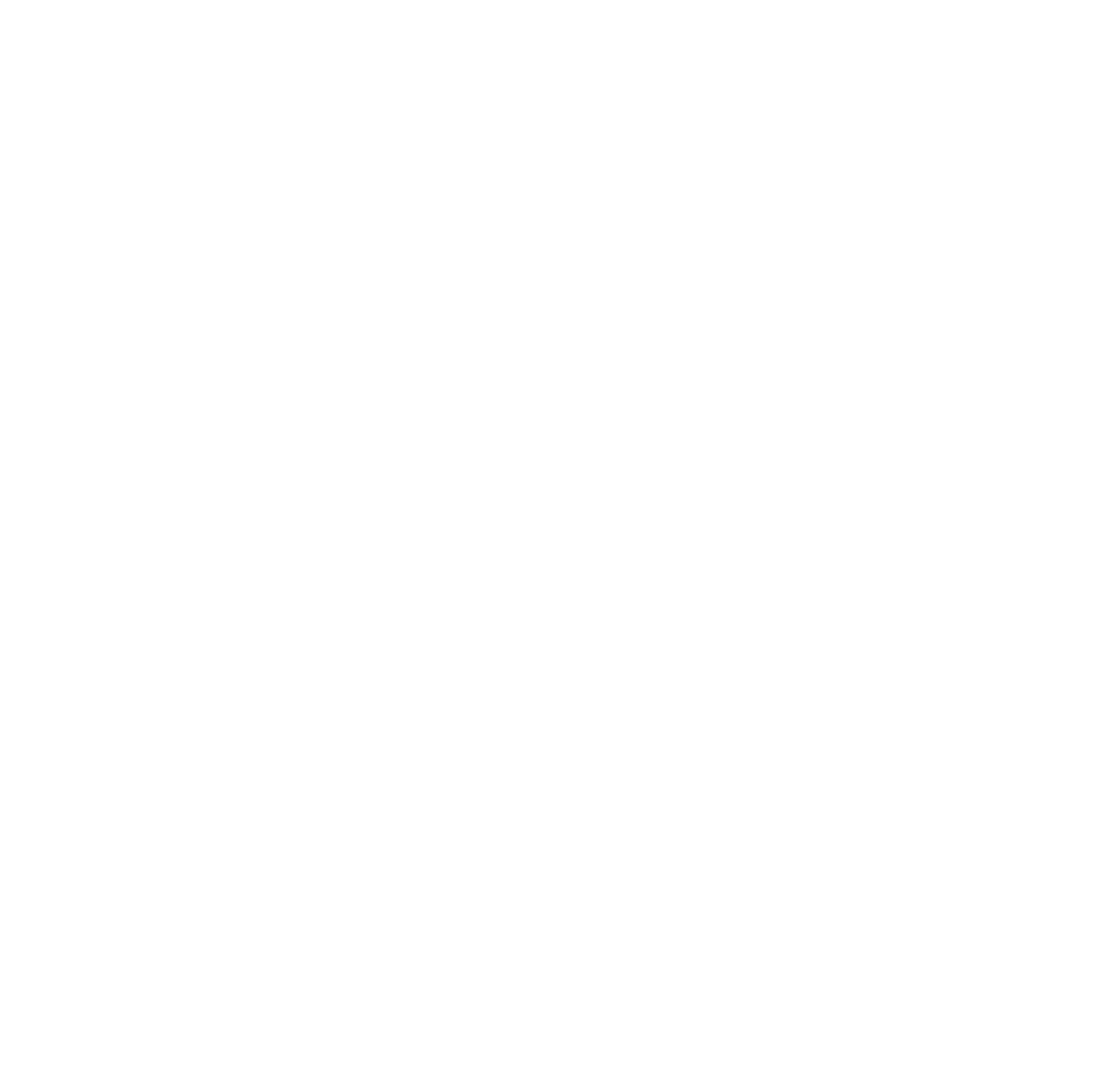

